\title{
A role for the claustrum in cognitive control
}

Maxwell B. Madden ${ }^{1}$, Brent W. Stewart ${ }^{1,2}$, Michael G. White ${ }^{1}$, Samuel R. Krimmel ${ }^{3}$, Houman Qadir $^{1}$, Frederick S. Barrett ${ }^{4}$, David A. Seminowicz ${ }^{2}$, and Brian N. Mathur ${ }^{1}$

\section{Author affiliations:}

1 Department of Pharmacology, School of Medicine, University of Maryland, Baltimore, MD, 21201, USA.

2 Department of Neural and Pain Sciences, School of Dentistry, and Center to Advance Chronic Pain Research, University of Maryland, Baltimore, MD, 21201, USA.

3 Department of Neurology, Washington University School of Medicine, St. Louis, MO 63110, USA

4 Department of Psychiatry and Behavioral Sciences, Johns Hopkins University School of Medicine, Baltimore, MD, 21224, USA.

Correspondence to:

Brian N. Mathur

HSF III RM 9-179

670 West Baltimore $\mathrm{St}$

Baltimore, MD 21201

Phone: 410-706-8239

Fax: 410-706-8341

bmathur@som.umaryland.edu

Running title: A role for the claustrum in cognitive control

Keywords: cognition; attention; working memory; cognitive network; cortex

Abbreviations: $\mathrm{ACC}=$ Anterior Cingulate Cortex $\mathrm{Cd}=$ Caudate $\mathrm{CON}=$ Cingulo-Opercular Network; DAN = Dorsal Attention Network; DMN = Default Mode Network; EVC = Expected Value of Control; fMRI = Functional Magnetic Resonance Imaging; FPN = Frontoparietal Network; IC = Insular Cortex; NICC = Network Instantiation in Cognitive Control; Pu = Putamen; $\mathrm{SN}=$ Salience Network; Str = Striatum; tACS = Transcranial Alternating Current Stimulation; $\mathrm{VAN}=$ Ventral Attention Network; WM = White Matter 


\begin{abstract}
Early hypotheses of claustrum function were fueled by neuroanatomical data and suggested that the claustrum is involved in processes ranging from salience detection to multisensory integration for perceptual binding. While these hypotheses spurred useful investigation, incompatibilities inherent in these views must be reconciled to further conceptualize claustrum function amid a wealth of new data. Here, we review the varied models of claustrum function and synthesize them with developments in the field to produce a new model of claustrum function: Network Instantiation in Cognitive Control (NICC). This model proposes that frontal cortices direct the claustrum to flexibly instantiate cortical networks to subserve cognitive control. We present literature support for this model and provide testable predictions arising from this conceptual framework.
\end{abstract}




\section{INTRODUCTION}

The claustrum is a thin, elongated sub-cortical telencephalic nucleus that possesses bidirectional connections with many areas of the cerebral cortex [1-13]. The claustrum is positioned between the insula and putamen and, in primates, is bounded by the external and extreme capsules (Figure 1). The claustrum appears in all therian mammals, at least some monotremes [14-16] and was recently discovered in reptiles [17]. The claustrum is composed of spiny glutaminergic projection neurons that exit claustrum to predominantly target cortex. In addition, multiple subpopulations of GABAergic, aspiny interneurons reside in the claustrum, differentiated by their expression of calcium binding proteins or neuropeptides, including parvalbumin [18-20], somatostatin, or vasoactive intestinal peptide [18].

The elongated shape of the claustrum and its proximity to white matter structures, dorsolateral striatum (caudate in primates) and insular cortex have historically limited the ability to specifically manipulate the claustrum. As a result, most early hypotheses of claustrum function are disproportionately based on structural data obtained from neuronal tract-tracing. However, the advent of neural circuit-specific approaches and whole-brain functional imaging has enabled a more direct assessment of claustrum function through recording of claustrum activity in the context of behavior and cognition. Utilizing this expanded access to the structure, the diverse field of claustrum hypotheses may now be revisited and refined.

Here we first describe previous hypotheses of claustrum function before advancing a new theory that unifies earlier concepts: specifically, that the claustrum receives pre-frontal cortical signals for coordinating the engagement of downstream cortical areas to meet cognitive demands. We call this model network instantiation in cognitive control (NICC). We describe evidence for the NICC model, examine the model's similarities and differences with other theories of claustrum function, and explain how predictions of the NICC model can be tested in future research (Box 1). 


\section{EXISTING FUNCTIONAL HYPOTHESES}

Models of claustrum function fall into three "families" of hypotheses that relate to: integration of cortical sensory information, salience and attentional processing, and cortical network dynamics. These hypotheses will be described in roughly the order they have been proposed.

\section{Integration}

Guided by an early claustrum lesion study in dogs in which auditory conditioning was impaired [21], along with observations of non-somatotopic multimodal responses in the claustrum, Spector, Hassmannova and Albe-Fessard [22] hypothesized that the claustrum associates sensory stimuli across modalities. The involvement of the claustrum in sensory processing was supported in an earlier study in curarized cat in which claustrum neuronal firing was observed in response to somatic nerve stimulation [23].

In an influential proposal, Ettlinger and Wilson [24] postulated that all areas of cortex have access to information from other (sensory) cortices through the claustrum, passing information through a series of cortico-claustro-cortical channels. In this way, the claustrum would function as a cross-modal transfer locus to support sensory integration. Crick and Koch [25] expanded upon the Ettlinger and Wilson [24] sensory integration model by proposing that the claustrum participates in perceptual binding by coordinating cortical processing. Perceptual binding refers to the process by which prior experience and ongoing sensory information gathered across multiple modalities are combined into a singular, integrated experience.

Following tract-tracing studies demonstrating widespread bi-directional claustrum connectivity with the cerebral cortex, Alloway, Smith, Beauchemin and Olson [26] proposed a somewhat divergent function compared to Crick and Koch. Alloway, Smith, Beauchemin and Olson [26] proposed a sensorimotor integration function which, at least in rodents, would serve to bilaterally coordinate whisker movement. This view introduced motor control as a possible outcome of claustrum-mediated sensory integration.

In an elaboration upon the "binding hypothesis" of Crick and Koch, Smythies, Edelstein and Ramachandran [27] proposed that the claustrum synchronizes oscillations from different 
cortical regions to give rise to binding. This hypothesis was further refined in Smythies, Edelstein and Ramachandran [28], which, similar to Crick and Koch [25], speculated about the existence of intra-claustral interactions and the possibility of dynamic cortico-claustro-cortical circuits that would serve as a mechanism of both regulating cortical synchrony and perceptual binding.

These iterations of the "binding hypothesis" rely upon claustrum neurons being responsive to multimodal sensory inputs. While a function for the claustrum in multimodal sensory processing is supported by early work in curarized cat [22,23] and during multimodal tasks in human positron emission tomography [29], it is challenged by later observations of unimodal responses to naturalistic stimuli in awake monkeys [30]. The "binding hypothesis" also suggests that the claustrum is integral in producing states of consciousness. However, in a study of five epilepsy patients, bilateral electrical stimulation of the claustrum did not result in loss or alteration of subjective awareness [31], and the claustrum remains relatively quiescent during a conscious resting state $[22,23,30,32]$. Despite a lack of strong evidence for the claustrum as a conductor of consciousness, these series of hypotheses generated core concepts that guided the emergence of a new generation of salience and attentional models of claustrum function.

\section{Salience Detection and/or Attention}

A role for the claustrum in salience processing for attention was proposed in 2008 [33]. This was prompted by anatomical data showing that the claustrum is strongly connected with anterior cingulate cortex (ACC), which is implicated in monitoring conflicts in decision making processing $[34,35]$ and is a component of the salience network [33, 36]. Remedios, Logothetis and Kayser [30] similarly suggested a salience detector function for the claustrum after observing unimodal transient claustrum activation in monkey in response to behaviorally relevant stimuli, such as conspecific vocalizations.

In an updated view, Mathur [37] suggested that the claustrum receives bottom-up signals from sensory and motor cortices and subsequently filters and propagates these salient signals to executive cortices, such as the ACC, to subserve bottom-up attention [33, 37]. Additionally, the claustrum was proposed to receive top-down signals from executive cortices (e.g. ACC) and relay them to sensory and motor areas to enhance processing of attended sensory modalities. This 
proposal placed the claustrum within bottom-up and top-down information processing frameworks.

Goll, Atlan and Citri [38] expanded upon the Mathur [37] hypothesis by proposing that the claustrum mediates the allocation of cortical processing for selective attention. In this model, claustro-cortical projections suppress cortical activity to reduce processing of unattended information. Thus, the claustrum is proposed as an "attentional searchlight", a term Crick [39] coined when discussing a similar function for the thalamic reticular nucleus.

Later, in response to functional magnetic resonance imaging (fMRI) observations of functional connectivity between the claustrum and insula, medial prefrontal cortex, and cingulate cortex in rodent, Smith, Liang, Watson, Alloway and Zhang [40] proposed that the claustrum integrates input from the basolateral amygdala, mediodorsal thalamus, and the medial prefrontal cortex to direct relevant cortical structures toward salient features of stimuli. Further, Smith, Watson, Liang, Liu, Zhang and Alloway [41] suggested that the claustrum participates in bottomup attentional processes: receiving salience information from limbic structures, then distributing that information to higher cortical areas, such as the frontal eye field, to ultimately result in cognitive and behavioral orientation to a stimulus; a function not unlike that proposed for the superior colliculus [42].

\section{CORTICAL NETWORKS AND COGNITIVE CONTROL}

The claustrum was recently proposed to play a role in cortical network switching [7]. In this section we will first give a brief overview of cortical networks and cognitive control, then more specifically describe how the claustrum fulfills a key mechanism of network interaction facilitating cognitive control. This lays the groundwork for the presentation of our new model of claustrum as a key hub modulating networks of cognitive control, presented in the next section.

Cognitive control is the ability to initiate, maintain, and monitor relevant information during goal-directed action, as opposed to habitual stimulus-response behavior [43, 44]. Cognitive control therefore demands flexible engagement of cognitive processes moment to moment. fMRI has helped reveal that these cognitive processes are not the domain of singular brain regions, but 
of networks, which are defined as collections of regions demonstrating coactivation during tasks and/or correlated activity at rest [45-47]. Despite substantial evidence for the emergence of cortical networks across species [45, 48-55], it remains unclear how non-neighboring cortical areas are synchronized for networked processing.

Over the last 30 years, neuroimaging studies accumulated identifying sets of cortical regions with correlated activity at rest [56-58]. This suggests that the brain possesses an intrinsic network architecture [59] that constrains the spatiotemporal organization and interaction of taskassociated networks [60]. More recently, several groups attempted to map the human brain's network organization more comprehensively [60-64]. Such parcellations are not identical, but they consistently include local sensory and somatomotor networks, as well as reproducible, geographically distributed networks of association cortices. A widely cited 7-network parcellation [61] of the human brain includes: visual and somatomotor networks; a default mode network (DMN) notable for increased metabolic activity at rest and decreased BOLD signal during externally focused tasks [58, 65]; a frontoparietal network (FPN) active during tasks; dorsal (DAN) and ventral (VAN) attention networks active during tasks; and a limbic network. More granular parcellations reveal additional networks, such as sub-components of the DMN [45], as well as salience (SN) and cingulo-opercular (CON) networks [57]. More broadly, a commonly used gross taxonomy divides the brain into two anti-correlated networks: a task-positive network active during tasks and a task-negative network, which largely overlaps with the DMN [59].

Several cortical networks possessing regions in the prefrontal cortex are associated with cognitive control [66]. The FPN [53] comprises regions associated with functions such as decisionmaking, maintaining task sets, and working memory. The DAN [67, 68] is "involved in preparing and applying goal-directed (top-down) selection for stimuli and responses" [68], while the VAN directs orientation toward salient stimuli in a bottom-up fashion [68]. The $\mathrm{SN}-$ as the name implies - activates in the presence of salient stimuli or thoughts [69], and the CON shows sustained activity related to maintaining and updating rules in a task [70]. While the $\mathrm{CON}$ and $\mathrm{SN}$ are distinct networks, they possess similar topography $[46,62]$.

Several models have been proposed for how these cortical networks interact to dynamically support cognitive states. They include the VAN updating the DAN in response to potentially 
important environmental sensory stimuli [68], the SN or CON performing the "switch" between task-negative and task-positive networks [36, 71, 72], and a dual-network model wherein a rapidadaptive FPN and a set-maintenance CON act in parallel. That is, the FPN quickly adapts to initiate and adjust cognitive control during a task while the CON, on a longer timescale, supports task set maintenance [73].

Graph theory analyses of whole brain imaging data have also yielded insights into how distributed brain systems may interact. Conceptualizing the brain as a collection of nodes connected by edges allows description of the brain in terms of communities of nodes varying in the number (nodal degree) and diversity (participation coefficient) of their connections. For example, Van Den Heuvel and Sporns [74] identified a collection of hub regions that not only possessed a high number of connections throughout the brain, but also demonstrated preferential connectivity among themselves. They postulated that this "rich club" of brain hubs may contribute to information integration. Bertolero, Yeo and D'Esposito [75] identified a largely distinct set of hubs, they labeled the "diverse club", notable for high levels of participation in multiple network communities. Importantly, task-dependent cortical network functional connectivity is modulated by hubs with a high participation coefficient [76], and these "diverse club" hubs exhibit substantial overlap with cognitive control-associated networks such as the FPN, DAN, and CON [75, 76].

\section{The claustrum as a cognitive control hub}

The claustrum possesses hallmark characteristics of a network hub. The claustrum has the highest connectivity per unit volume in the brain [10]. Similarly to "diverse" and "rich" hubs [74, 75], the effects of removing claustral connections from network graphs suggest they efficiently link multiple brain networks [10]. Also, in many brain disorders, abnormalities are more likely to be found in hub regions than elsewhere [77]. Accordingly, claustrum abnormalities are associated with a variety of neuropsychiatric symptoms $[10,78]$, including difficulties in cognitive control [79].

The claustrum also interacts considerably with cognitive control regions. It shares its densest structural connectivity with frontal cortices [10], which anchor cognitive control networks [66], and is bidirectionally connected to prefrontal regions exhibiting significant evolutionary 
expansion that are associated with higher-order cognition [7]. Furthermore, the claustrum receives strong input from the ACC $[6,11,12,80]$, a key node in the SN and CON, which has been placed within a "core system" for cognitive control [71, 81]. For example, Shenhav, Botvinick and Cohen [81] proposed that the dorsal ACC is responsible for determining the expected value of control (EVC): defined as an evaluation of the potential benefits of exerting cognitive control against the potential costs. Thus, the claustrum is situated to receive such cognitive control signals and may serve as a hub through which prefrontal cortical regions direct dynamic interplay of cortical networks to optimize cognitive control. In the next section, we describe a specified model for claustrum function that expands on this concept.

\section{THE NETWORK INSTANTIATION IN COGNITIVE CONTROL (NICC) MODEL}

We propose that the claustrum serves a cognitive control function by instantiating a cortical network with appropriate network integrity for optimal cognitive performance. To accomplish this, the NICC model proposes that the claustrum: 1) receives a signal from frontal cortices to prompt emergence of a specific network (a network initiation signal); 2) transforms and amplifies this signal and; 3) broadcasts the transformed signal through return projections to frontal cortices and

projections to posterior sensory and association cortices to disrupt prior processing and bring targeted cortical cognitive network nodes into phase (Figure 2). This allows other inputs (such as from thalamus) to set oscillatory frequency and continue maintenance of synchronization. We view this process as required for implementation of goal-directed behavior under cognitive conflict and/or high cognitive demand.

\section{Structural and functional connectivity}

During task engagement, frontal cortical activation precedes activation of posterior cortex in macaques [82, 83], and in humans frontal cortical activity precedes network emergence [84, 85]. Frontal cortical input to the mouse claustrum is substantially stronger than input from posterior sensory and sensory association cortices [11, 13, 86, 87]. For instance, while optogenetic 
stimulation of frontal input (e.g. ACC) readily drives action potentials in claustrum projection neurons in mouse acute brain slices, stimulation of input from various combinations of sensory cortical inputs fail to produce claustrum firing [86]. Claustrum neurons projecting to posterior parietal association and visual cortices are among those that fire in response to ACC afferent stimulation [88]. The rodent claustrum also provides dense innervation to frontal areas, including the ACC and the prelimbic prefrontal cortex [11, 12]. Finally, claustrum output to cortex may differ based on the source of claustrum input as multiple input-specific pathways of information through the claustrum exist [89]. Together, these data support a neural circuit mechanism for frontal cortical areas to communicate, through the claustrum, to specific downstream cortical nodes composing a network (Figure 2).

The connectivity necessary for the claustrum to support cortical network nodes is observed in monkey as well. The claustrum projects to dorsolateral PFC in capuchin monkey [4, 7] and posterior parietal cortex in capuchin and macaque monkeys [90]. These regions are key nodes of the FPN $[68,85,91,92]$. With regards to task-negative networks, the claustrum bidirectionally connects with posterior cingulate cortex in both the Crab-eating and rhesus macaque [93], receives projections from the inferior parietal lobule in stump-tailed monkey [94], and projects to entorhinal cortex in macaque $[95,96]$. The claustrum projects to the frontal pole in capuchin and macaque monkeys $[7,97]$ and, in macaque, these projections compose up to $50 \%$ of subcortical input to the frontal pole [97]. The frontal pole additionally sends projections to the claustrum [98]. The claustrum also projects to Brodmann areas 24b/c in capuchin monkey [7], a region of dorsomedial prefrontal cortex. The inferior parietal lobule, entorhinal cortex, ventromedial prefrontal cortex (which includes frontal pole) and dorsomedial prefrontal cortex are established nodes of the DMN [99-101].

Functional connectivity in humans, as assessed by fMRI, mirrors structural connectivity seen in rodent and monkey data. The human claustrum is functionally connected to both taskpositive and task-negative networks $[102,103]$. High expression of serotonin 2A receptor in the claustrum [104-110], the primary target for the psychedelic drug psilocybin, suggests the claustrum may participate in cognitive network disruption by psychedelic drugs. Indeed, psilocybin administration in humans decreases right claustrum functional connectivity with DMN 
and increases functional connectivity with FPN, and the integrity of both DMN and FPN networks is associated with functional connectivity with the right claustrum [103].

Together, data from these structural and functional connectivity studies position the claustrum to support networks comprised of both frontal and posterior cortical areas, and sets the stage for co-activation of these spatially distant cortical areas by the claustrum in response to a top-down frontal cortical signal. Next, we will present mechanistic explanations based on extant data for how the claustrum responds to top-down frontal cortical signals and how the claustrum may, in turn, participate in bringing cortices into phase to facilitate synchronization for network instantiation [111].

\section{Claustrum responsivity to top-down cortical input}

If claustrum projection neurons initiate synchronization of constellations of cortical areas to give rise to task-relevant networks, then the activity of claustrum neurons would be expected to activate: 1) at the initiation of a task and, therefore, at network instantiation; 2) in a manner that briefly amplifies incoming signals; 3) faithfully in response to frontal input and otherwise remain quiescent.

\section{Claustrum activity at task-initiation}

Mouse ACC input activity and the activity of the claustrum itself increases prior to the presentation of a cue in the cognitively demanding five choice serial response time task [86, 112]. Photoinhibition of either ACC afferents to the claustrum, or the claustrum itself, diminishes task performance in mouse [86, 112]. Conversely, inhibition of claustrum, or ACC afferents, during

performance of the less demanding one choice serial response time task does not impair performance [86, 112]. In rodent, blanket claustrum inhibition decreases performance in both pupretrieval and two-alternative forced-choice tasks, but only in the presence of an auditory distractor [113]. In rabbit, the claustrum is necessary for the optimal acquisition of classically conditioned eyeblink responses, but not the expression of these responses once they are learned [32]. In humans, claustrum activity is significantly greater than baseline only at the onset of difficult versions of the multi-source interference task [102]. This activity peak occurs when task-positive 
networks emerge and the DMN disengages [102]. These data support the notion that the claustrum activates at the initiation of task-positive networks and is necessary for optimal performance in tasks requiring those networks.

\section{Claustrum amplification of input signals}

An ideal network instantiation signal must be both powerful, to interrupt any previous cortical processing, and transient, to prevent interference with subsequent task-relevant processing. The claustrum possesses internal microcircuitry that allows the shaping of cortical inputs into transiently amplified claustro-cortical outputs. To produce amplification of frontal input signals, roughly half of claustrum projection neurons respond to input non-linearly; these neurons burst fire disproportionately to cortical input strength $[18,20]$. On average across all claustrum projection neuron types, optogenetic activation of ACC inputs at 1-10 Hz input frequencies produce $50-80 \mathrm{~Hz}$ claustrum projection neuron firing frequencies [86]. This amplified output firing is transient; lasting a few seconds before exponentially decaying to below input frequencies [20]. Additionally, cortical inputs to the claustrum innervate both claustrum projection neurons and parvalbumin-positive interneurons, the latter of which provides a classical feedforward microcircuit inhibition onto claustrum projection neurons that constrain their neuron firing [86, 114]. This corresponds with observations of claustrum neuron firing transience in monkey $(\sim 0.2$ seconds) in vivo [115] as well as in mouse acute brain slice, where a less than one second firing period in claustrum neurons is observed in response to a five second, $20 \mathrm{~Hz}$ optogenetic stimulation of ACC afferents [86, 88].

\section{Faithful claustrum responsivity to frontal cortical input over quiescent background activity}

In acute brain slices, claustrum projection neurons readily fire in response to excitatory input from frontal cortices, which is in stark contrast to the inability for claustrum neurons to fire in response to activation of sensorimotor cortical inputs [20]. Generally, claustrum firing in the absence of a stimulus is sparse, as commonly noted across in vivo electrophysiological recordings in cat [22, 23], rabbit [32], and monkey [30]. Providing a possible mechanism for this quiescence, local parvalbumin-positive interneurons have high electrical interconnectivity [114] and strongly 
innervate claustrum projection neurons [86, 114], thereby suppressing spontaneous claustrum activity in the absence of cortical input activity (Figure 4).

\section{Claustrum participation in synchronization of cortex}

The NICC model proposes that the claustrum brings cortical activity into phase to instantiate a distributed network (Box 2). This proposal is supported by existing evidence: the claustrum coordinates synchronous cortical slow-wave activity in awake mice by producing a sustained cortical down-state [116], which is similar to the sustained cortical suppression observed by Jackson, Karnani, Zemelman, Burdakov and Lee [117] in awake head-fixed mice. Furthermore, the claustrum generates synchronous sharp waves in slow-wave sleep in reptiles [17]. These studies establish the potential ability of the claustrum to synchronize cortex for network initiation in waking states.

To achieve cross-cortical synchrony, the NICC model proposes that the claustrum activates cortical inhibitory microcircuits to achieve cross-cortical down states, from which synchronous activation emerges. Jackson, Karnani, Zemelman, Burdakov and Lee [117] showed that in awake mice, optogenetic activation of claustrum input to PFC transiently inhibits net cortical pyramidal neuron activity by activating cortical neuropeptide $Y$ positive interneurons. This cortical inhibition is followed by a resumption of excitation, transiently driving pyramidal neuron activity above baseline levels $[117,118]$. The NICC model proposes that when this occurs simultaneously across disparate areas of cortex, it allows network instantiation by inducing a temporally phase-locked cortical up-state. From this up-state, other inputs to cortex may sustain cortical oscillations

underlying network activity (Figure 3). Supporting the notion that claustro-cortical signaling may override ongoing activity, optogenetic activation of claustro-cortical neurons results in greater inhibition of mouse PFC neuron firing in vivo than all other major subcortical inputs [117].

\section{Similarities and Differences with Previous Models}

The NICC model integrates the current literature on claustrum function, thus updating and advancing previous models, and provides testable circuit-based mechanisms. Advancing the proposals by Crick and Koch [25] and, later, Smythies, Edelstein and Ramachandran [28] that the 
claustrum coordinates widespread cortical areas, the NICC model attributes this cortical coordination to a cognitive control function rather than generating conscious percepts. The NICC model proposes that the claustrum distributes top-down cognitive control signals, rather than integrating information from sensory cortices for perceptual binding. Advancing the model proposed by Vidyasagar and Levichkina [119], in which claustrum enhances cortical synchrony by passing low frequency "cross frequency coupling" signals between cortical areas, the NICC model predicts the claustrum's role in this synchrony is in "preparing" cortex for synchronized oscillatory activity that is supported by other inter-cortical connections or thalamocortical connections [119-125]. Advancing the proposals by Goll, Atlan and Citri [38] and later Jackson, Karnani, Zemelman, Burdakov and Lee [117] that the claustrum achieves top-down control of cortex by suppressing activity in unattended modalities to serve a selective attention, or attentional "spotlight", role, the NICC model instead proposes that the claustrum inhibits cortex so that cortical area will re-activate in synchrony with other select cortical areas. Finally, advancing the proposal by Jackson, Smith and Lee [118] that claustrum feed-forward inhibition of cortex combined with the bifurcation of claustro-cortical axons to innervate different cortical layers and regions could serve to synchronize spike timing and facilitate communication between different cortical areas, the NICC model proposes that the claustrum does not aid in the detection or processing of salient information per se, but does assist in mobilizing cortical structures for responses to salient stimuli that are task-relevant.

The NICC model of claustrum function stands as both distinct and synergistic with that of other possible circuit-based mechanisms of cortical network support, including cortico-thalamocortical and cortico-cortical processing. Thalamic nuclei, such as mediodorsal nucleus, pulvinar, and thalamic reticular nucleus, each uniquely regulate cortical processing. In contrast to the claustrum, thalamic mediodorsal nucleus input to prefrontal cortex continues throughout a task [125] and appears to function to sustain attention on the task by amplifying local prefrontal cortex connectivity [124]. The pulvinar nucleus mediates synchrony between neighboring cortical regions [121] and is implicated in task-switching within the visual domain [126]. Unlike the claustrum, and similarly to mediodorsal nucleus, the pulvinar is highly active, consistently exchanging signals with cortex [126]. The thalamic reticular nucleus functions as a "neuronal oscillator" [123] that determines the frequency of cortical rhythms [122] and is implicated in 
selective attention similar to the "searchlight" hypotheses proposed by Crick [39, 127, 128]. Ultimately, both the claustrum and thalamic nuclei must bear separate but mutually dependent roles in cortical processing for cognition; claustrum functioning to prepare cortex for networked activity, while thalamic nuclei may directly participate in ongoing functional cortical connectivity. Finally, cortico-cortical circuits are proposed to support network architecture. For example, the mouse DMN, as defined by fMRI analysis, is composed of cortical regions that are preferentially connected through layer $2 / 3$ cortico-cortical connections with one another. This finding provides a potential circuit mechanism for within-network maintenance. Future investigation should therefore test predictions of the NICC model within the context of the distributed network of extended cortical systems (Box 1).

The NICC model provides testable circuit-based mechanisms for existing cognitive control functions. For example, the NICC model can be used to test existing models of network switching. Behaviorally, the NICC model proposes a function for the claustrum in cognitive control that is distinct from bottom-up salience detection, which activates the SN. Structurally, the claustrum is highly bidirectionally connected with the ACC [129], a central node of the SN [130, 131], but it receives few, if any, projections from anterior insula [129], the other key SN node [36, 69, 130]. Anterior insula activation predicts FPN activation and DMN deactivation [132] and anterior insula dysfunction is associated with impaired network switching [133], leading to the proposal that SN mediates network switching [36]. The NICC model is situated to test predictions of this model, wherein the anterior insula (encoding salience detection) may signal to the ACC (encoding attention selection [134]) which, in turn, signals to the claustrum to initiate the synchrony of networks supporting cognitive processing. Thus, the NICC model functions as a unique conceptual framework from which to derive circuit-level, testable predictions of network activity in cognitive control (Box 1).

\section{CONCLUSION}

The NICC model posits that the claustrum instantiates cortical networks through synchronization of cortical network nodes in a manner commensurate with the cognitive demand of a given task. This function is achieved in three parts: the claustrum receives frontal cortical task demand 
information, transforms and amplifies this signal, and finally synchronizes targeted cortical areas to instantiate a cognitive network. This model reflects the current status of data from the claustrum field, which greatly requires further expansion. Inherent in the NICC model is a proposal of the claustrum mediating cognitive control, which is subject to dysfunction in a range of neuropsychiatric disorders [135-139]. If further supported, the claustrum represents a new node for further understanding of cognition.

\section{Funding}

This work was supported by the US National Institute on Alcohol Abuse and Alcoholism grant R01AA024845 (B.N.M.).

\section{Competing Interests}

The authors report no competing interests.

\section{References}

1. Macchi, G. et al. (1981) The organization of the claustroneocortical projections in the cat studied by means of the HRP retrograde axonal transport. Journal of Comparative Neurology 195 (4), 681-695.

2. Baizer, J. et al. (1997) Projections from the claustrum to the prelunate gyrus in the monkey. Experimental brain research $113(3), 564-568$.

3. Sadowski, M. et al. (1997) Rat's claustrum shows two main cortico-related zones. Brain research 756 (12), 147-152.

4. Tanné-Gariépy, J. et al. (2002) Projections of the claustrum to the primary motor, premotor, and prefrontal cortices in the macaque monkey. Journal of Comparative Neurology 454 (2), 140-157.

5. Hur, E.E. and Zaborszky, L. (2005) Vglut2 afferents to the medial prefrontal and primary somatosensory cortices: a combined retrograde tracing in situ hybridization. Journal of Comparative Neurology 483 (3), 351-373.

6. Reser, D.H. et al. (2017) Topography of claustrum and insula projections to medial prefrontal and anterior cingulate cortices of the common marmoset (Callithrix jacchus). J Comp Neurol 525 (6), 1421-1441.

7. Reser, D.H. et al. (2014) Claustrum projections to prefrontal cortex in the capuchin monkey (Cebus apella). Frontiers in systems neuroscience 8, 123.

8. Milardi, D. et al. (2015) Cortical and subcortical connections of the human claustrum revealed in vivo by constrained spherical deconvolution tractography. Cereb Cortex 25 (2), 406-14.

9. Arrigo, A. et al. (2017) Inter-hemispheric Claustral Connections in Human Brain: A Constrained Spherical Deconvolution-Based Study. Clin Neuroradiol 27 (3), 275-281. 
10. Torgerson, C.M. et al. (2015) The DTI connectivity of the human claustrum. Human brain mapping 36 (3), 827-838.

11. Wang, Q. et al. (2017) Organization of the connections between claustrum and cortex in the mouse. Journal of Comparative Neurology 525 (6), 1317-1346.

12. White, M.G. et al. (2017) Cortical hierarchy governs rat claustrocortical circuit organization. J Comp Neurol 525 (6), 1347-1362.

13. Zingg, B. et al. (2018) Input-output organization of the mouse claustrum. J Comp Neurol 526 (15), 24282443.

14. Butler, A.B. et al. (2002) Apparent absence of claustrum in monotremes: implications for forebrain evolution in amniotes. Brain, Behavior and Evolution 60 (4), 230-240.

15. Ashwell, K.W. et al. (2004) The claustrum is not missing from all monotreme brains. Brain, behavior and evolution 64 (4), 223-241.

16. Suarez, R. et al. (2018) A pan-mammalian map of interhemispheric brain connections predates the evolution of the corpus callosum. Proc Natl Acad Sci U S A 115 (38), 9622-9627.

17. Norimoto, H. et al. (2020) A claustrum in reptiles and its role in slow-wave sleep. Nature 578 (7795), 413-418.

18. Graf, M. et al. (2020) Identification of mouse claustral neuron types based on their intrinsic electrical properties. eNeuro.

19. Brown, S.P. et al. (2017) New Breakthroughs in Understanding the Role of Functional Interactions between the Neocortex and the Claustrum. J Neurosci 37 (45), 10877-10881.

20. White, M.G. and Mathur, B.N. (2018) Claustrum circuit components for top-down input processing and cortical broadcast. Brain Struct Funct 223 (9), 3945-3958.

21. Chorazyna, H. et al. (1965) Changes in auditory conditioning in dogs after lesions of the claustrum. Proc XXIII Int Cong Physiol Sci Tokyo 13, 456-457.

22. Spector, I. et al. (1974) Sensory properties of single neurons of cat's claustrum. Brain Research 66 (1), 39-65.

23. Segundo, J. and Machne, X. (1956) Unitary responses to afferent volleys in lenticular nucleus and claustrum. Journal of neurophysiology 19 (4), 325-339.

24. Ettlinger, G. and Wilson, W. (1990) Cross-modal performance: behavioural processes, phylogenetic considerations and neural mechanisms. Behavioural brain research 40 (3), 169-192.

25. Crick, F.C. and Koch, C. (2005) What is the function of the claustrum? Philosophical Transactions of the Royal Society B: Biological Sciences 360 (1458), 1271-1279.

26. Alloway, K.D. et al. (2009) Bilateral projections from rat MI whisker cortex to the neostriatum, thalamus, and claustrum: forebrain circuits for modulating whisking behavior. Journal of Comparative Neurology 515 (5), 548-564.

27. Smythies, J. et al. (2012) The Functional Anatomy of the Claustrum: The Net That Binds.

28. Smythies, J.R. et al. (2014) Hypotheses relating to the function of the claustrum. In The Claustrum, pp. 299-352, Elsevier.

29. Hadjikhani, N. and Roland, P.E. (1998) Cross-modal transfer of information between the tactile and the visual representations in the human brain: a positron emission tomographic study. Journal of Neuroscience 18 (3), 1072-1084.

30. Remedios, R. et al. (2010) Unimodal responses prevail within the multisensory claustrum. Journal of Neuroscience 30 (39), 12902-12907. 
31. Bickel, S. and Parvizi, J. (2019) Electrical stimulation of the human claustrum. Epilepsy Behav 97, 296303.

32. Reus-García, M. et al. (2020) The Claustrum is Involved in Cognitive Processes Related to the Classical Conditioning of Eyelid Responses in Behaving Rabbits. Cerebral Cortex.

33. Mathur, B.N., Unraveling the seat of consciousness: anatomical redefinition and molecular characterization of the claustrum, Vanderbilt University, 2008.

34. Botvinick, M.M. (2007) Conflict monitoring and decision making: reconciling two perspectives on anterior cingulate function. Cognitive, Affective, \& Behavioral Neuroscience 7 (4), 356-366.

35. Carter, C.S. and Van Veen, V. (2007) Anterior cingulate cortex and conflict detection: an update of theory and data. Cognitive, Affective, \& Behavioral Neuroscience 7 (4), 367-379.

36. Menon, V. and Uddin, L.Q. (2010) Saliency, switching, attention and control: a network model of insula function. Brain Structure and Function 214 (5-6), 655-667.

37. Mathur, B.N. (2014) The claustrum in review. Frontiers in systems neuroscience 8, 48.

38. Goll, Y. et al. (2015) Attention: the claustrum. Trends in neurosciences 38 (8), 486-495.

39. Crick, F. (1984) Function of the thalamic reticular complex: the searchlight hypothesis. Proceedings of the National Academy of Sciences 81 (14), 4586-4590.

40. Smith, J.B. et al. (2017) Interhemispheric resting-state functional connectivity of the claustrum in the awake and anesthetized states. Brain Struct Funct 222 (5), 2041-2058.

41. Smith, J.B. et al. (2019) A Role for the Claustrum in Salience Processing? Front Neuroanat 13, 64.

42. Basso, M.A. et al. (2021) Unraveling circuits of visual perception and cognition through the superior colliculus. Neuron.

43. Gratton, G. et al. (2018) Dynamics of cognitive control: Theoretical bases, paradigms, and a view for the future. Psychophysiology 55 (3), e13016.

44. Botvinick, M.M. et al. (2001) Conflict monitoring and cognitive control. Psychological review 108 (3), 624.

45. Buckner, R.L. and DiNicola, L.M. (2019) The brain's default network: updated anatomy, physiology and evolving insights. Nature Reviews Neuroscience 20 (10), 593-608.

46. Gratton, C. et al. (2018) Functional brain networks are dominated by stable group and individual factors, not cognitive or daily variation. Neuron 98 (2), 439-452. e5.

47. Bressler, S.L. and Menon, V. (2010) Large-scale brain networks in cognition: emerging methods and principles. Trends in cognitive sciences 14 (6), 277-290.

48. Chuang, K.-H. and Nasrallah, F.A. (2017) Functional networks and network perturbations in rodents. Neuroimage 163, 419-436.

49. Lu, H. et al. (2012) Rat brains also have a default mode network. Proceedings of the National Academy of Sciences 109 (10), 3979-3984.

50. Gozzi, A. and Schwarz, A.J. (2016) Large-scale functional connectivity networks in the rodent brain. Neuroimage 127, 496-509.

51. Arsenault, J.T. et al. (2018) Attention shifts recruit the monkey default mode network. Journal of Neuroscience 38 (5), 1202-1217.

52. Vincent, J.L. et al. (2007) Intrinsic functional architecture in the anaesthetized monkey brain. Nature 447 (7140), 83-86. 
53. Vincent, J.L. et al. (2008) Evidence for a frontoparietal control system revealed by intrinsic functional connectivity. Journal of neurophysiology 100 (6), 3328-3342.

54. Bressler, S.L. (1995) Large-scale cortical networks and cognition. Brain Research Reviews 20 (3), 288304.

55. Mantini, D. et al. (2011) Default mode of brain function in monkeys. Journal of Neuroscience 31 (36), 12954-12962.

56. Biswal, B. et al. (1995) Functional connectivity in the motor cortex of resting human brain using echoplanar MRI. Magnetic resonance in medicine 34 (4), 537-541.

57. Power, J.D. et al. (2014) Studying brain organization via spontaneous fMRI signal. Neuron 84 (4), 681 696.

58. Greicius, M.D. et al. (2003) Functional connectivity in the resting brain: a network analysis of the default mode hypothesis. Proceedings of the National Academy of Sciences 100 (1), 253-258.

59. Fox, M.D. et al. (2005) The human brain is intrinsically organized into dynamic, anticorrelated functional networks. Proceedings of the National Academy of Sciences 102 (27), 9673-9678.

60. Cole, M.W. et al. (2014) Intrinsic and task-evoked network architectures of the human brain. Neuron 83 (1), 238-251.

61. Thomas Yeo, B. et al. (2011) The organization of the human cerebral cortex estimated by intrinsic functional connectivity. Journal of neurophysiology 106 (3), 1125-1165.

62. Power, J.D. et al. (2011) Functional network organization of the human brain. Neuron 72 (4), 665-678.

63. Glasser, M.F. et al. (2016) A multi-modal parcellation of human cerebral cortex. Nature 536 (7615), 171-178.

64. Gordon, E.M. et al. (2017) Precision functional mapping of individual human brains. Neuron 95 (4), 791 807. e7.

65. Spreng, R.N. et al. (2010) Default network activity, coupled with the frontoparietal control network, supports goal-directed cognition. Neuroimage 53 (1), 303-317.

66. Menon, V. and D'Esposito, M. (2021) The role of PFC networks in cognitive control and executive function. Neuropsychopharmacology, 1-14.

67. Szczepanski, S.M. et al. (2013) Functional and structural architecture of the human dorsal frontoparietal attention network. Proceedings of the National Academy of Sciences 110 (39), 15806-15811.

68. Corbetta, M. and Shulman, G.L. (2002) Control of goal-directed and stimulus-driven attention in the brain. Nature reviews neuroscience 3 (3), 201-215.

69. Seeley, W.W. et al. (2007) Dissociable intrinsic connectivity networks for salience processing and executive control. Journal of Neuroscience 27 (9), 2349-2356.

70. Dosenbach, N.U. et al. (2007) Distinct brain networks for adaptive and stable task control in humans. Proceedings of the National Academy of Sciences 104 (26), 11073-11078.

71. Dosenbach, N.U. et al. (2006) A core system for the implementation of task sets. Neuron 50 (5), 799812.

72. Wu, T. et al. (2019) Anterior insular cortex is a bottleneck of cognitive control. Neurolmage 195, 490504.

73. Dosenbach, N.U. et al. (2008) A dual-networks architecture of top-down control. Trends in cognitive sciences $12(3), 99-105$.

74. Van Den Heuvel, M.P. and Sporns, O. (2011) Rich-club organization of the human connectome. Journal of Neuroscience 31 (44), 15775-15786. 
75. Bertolero, M.A. et al. (2017) The diverse club. Nature communications 8 (1), 1-11.

76. Gratton, C. et al. (2016) Evidence for two independent factors that modify brain networks to meet task goals. Cell reports 17 (5), 1276-1288.

77. Crossley, N.A. et al. (2014) The hubs of the human connectome are generally implicated in the anatomy of brain disorders. Brain 137 (8), 2382-2395.

78. Zuhorn, F. et al. (2021) Parainfectious encephalitis in COVID-19:"the claustrum sign". Journal of neurology 268 (6), 2031-2034.

79. Sener, R. (1993) The claustrum on MRI: normal anatomy, and the bright claustrum as a new sign in Wilson's disease. Pediatric radiology 23 (8), 594-596.

80. Vogt, B.A. et al. (1979) Thalamic and cortical afferents differentiate anterior from posterior cingulate cortex in the monkey. Science 204 (4389), 205-207.

81. Shenhav, A. et al. (2013) The expected value of control: an integrative theory of anterior cingulate cortex function. Neuron 79 (2), 217-240.

82. Buschman, T.J. and Miller, E.K. (2007) Top-down versus bottom-up control of attention in the prefrontal and posterior parietal cortices. science 315 (5820), 1860-1862.

83. Gregoriou, G.G. et al. (2009) High-frequency, long-range coupling between prefrontal and visual cortex during attention. science 324 (5931), 1207-1210.

84. Grent, T. and Woldorff, M.G. (2007) Timing and sequence of brain activity in top-down control of visualspatial attention. PLoS Biol 5 (1), e12.

85. Li, L. et al. (2010) Role of frontal and parietal cortices in the control of bottom-up and top-down attention in humans. Brain research 1344, 173-184.

86. White, M.G. et al. (2018) Anterior Cingulate Cortex Input to the Claustrum Is Required for Top-Down Action Control. Cell Rep 22 (1), 84-95.

87. Smith, J.B. and Alloway, K.D. (2010) Functional specificity of claustrum connections in the rat: interhemispheric communication between specific parts of motor cortex. Journal of Neuroscience 30 (50), $16832-16844$.

88. White, M.G. and Mathur, B.N. (2018) Frontal cortical control of posterior sensory and association cortices through the claustrum. Brain Struct Funct 223 (6), 2999-3006.

89. Chia, Z. et al. (2020) Synaptic Connectivity between the Cortex and Claustrum Is Organized into Functional Modules. Current Biology.

90. Leichnetz, G.R. (2001) Connections of the medial posterior parietal cortex (area $7 \mathrm{~m}$ ) in the monkey. The Anatomical Record: An Official Publication of the American Association of Anatomists 263 (2), 215236.

91. Ungerleider, S.K. and G, L. (2000) Mechanisms of visual attention in the human cortex. Annual review of neuroscience 23 (1), 315-341.

92. Fan, J. et al. (2005) The activation of attentional networks. Neuroimage 26 (2), 471-479.

93. Baleydier, C. and Mauguiere, F. (1980) The duality of the cingulate gyrus in monkey. Neuroanatomical study and functional hypothesis. Brain: a journal of neurology 103 (3), 525.

94. Weber, J.T. and Yin, T.C. (1984) Subcortical projections of the inferior parietal cortex (area 7) in the stump-tailed monkey. Journal of Comparative Neurology 224 (2), 206-230.

95. Insausti, R. et al. (1987) The entorhinal cortex of the monkey: III. Subcortical afferents. Journal of Comparative Neurology 264 (3), 396-408. 
96. Mufson, E.J. and Mesulam, M.M. (1982) Insula of the old world monkey. II: Afferent cortical input and comments on the claustrum. Journal of Comparative Neurology 212 (1), 23-37.

97. Burman, K.J. et al. (2011) Subcortical projections to the frontal pole in the marmoset monkey. European Journal of Neuroscience 34 (2), 303-319.

98. Petrides, M. and Pandya, D.N. (2007) Efferent association pathways from the rostral prefrontal cortex in the macaque monkey. Journal of Neuroscience 27 (43), 11573-11586.

99. Sheline, Y.I. et al. (2009) The default mode network and self-referential processes in depression. Proceedings of the National Academy of Sciences 106 (6), 1942-1947.

100. Buckner, R.L. et al. (2008) The brain's default network: anatomy, function, and relevance to disease.

101. Raichle, M.E. et al. (2001) A default mode of brain function. Proceedings of the National Academy of Sciences 98 (2), 676-682.

102. Krimmel, S.R. et al. (2019) Resting state functional connectivity and cognitive task-related activation of the human claustrum. Neuroimage 196, 59-67.

103. Barrett, F.S. et al. (2020) Psilocybin acutely alters the functional connectivity of the claustrum with brain networks that support perception, memory, and attention. Neuroimage 218, 116980.

104. Pasqualetti, M. et al. (1996) Comparative anatomical distribution of serotonin 1A, 1Da and 2A receptor mRNAs in human brain postmorten. Molecular brain research 39 (1-2), 223-233.

105. Pompeiano, M. et al. (1994) Distribution of the serotonin 5-HT2 receptor family mRNAs: comparison between 5-HT2A and 5-HT2C receptors. Molecular Brain Research 23 (1-2), 163-178.

106. Hamada, S. et al. (1998) Localization of 5-HT2A receptor in rat cerebral cortex and olfactory system revealed by immunohistochemistry using two antibodies raised in rabbit and chicken. Molecular brain research 54 (2), 199-211.

107. López-Giménez, J.F. et al. (1997) Selective visualization of rat brain 5-HT2A receptors by autoradiography with [3H] MDL 100,907. Naunyn-Schmiedeberg's archives of pharmacology 356 (4), 446454.

108. López-Giménez, J.F. et al. (2001) Mapping of 5-HT2A receptors and their mRNA in monkey brain:[3H] MDL100, 907 autoradiography and in situ hybridization studies. Journal of Comparative Neurology 429 (4), 571-589.

109. Zea-Ponce, Y. et al. (2002) Pharmacokinetics and brain distribution in non human primate of $R(-)[$ sup 123\} I] DOI, A 5HT \{sub 2A/2C\} serotonin agonist.

110. Hall, $\mathrm{H}$. et al. (2000) Autoradiographic localization of $5-\mathrm{HT} 2 \mathrm{~A}$ receptors in the human brain using [3H] M100907 and [11C] M100907. Synapse 38 (4), 421-431.

111. Raut, R.V. et al. (2021) Global waves synchronize the brain's functional systems with fluctuating arousal. Science advances 7 (30), eabf2709.

112. White, M.G. et al. (2020) The Mouse Claustrum Is Required for Optimal Behavioral Performance Under High Cognitive Demand. Biological Psychiatry.

113. Atlan, G. et al. (2018) The Claustrum Supports Resilience to Distraction. Curr Biol 28 (17), 27522762.e7.

114. Kim, J. et al. (2016) Synaptic Organization of the Neuronal Circuits of the Claustrum. J Neurosci 36 (3), 773-84.

115. Remedios, R. et al. (2014) A role of the claustrum in auditory scene analysis by reflecting sensory change. Frontiers in systems neuroscience 8, 44. 
116. Narikiyo, K. et al. (2020) The claustrum coordinates cortical slow-wave activity. Nat Neurosci 23 (6), 741-753.

117. Jackson, J. et al. (2018) Inhibitory Control of Prefrontal Cortex by the Claustrum. Neuron 99 (5), 10291039.e4.

118. Jackson, J. et al. (2020) The Anatomy and Physiology of Claustrum-Cortex Interactions. Annu Rev Neurosci.

119. Vidyasagar, T.R. and Levichkina, E. (2019) An Integrated Neuronal Model of Claustral Function in Timing the Synchrony Between Cortical Areas. Front Neural Circuits 13, 3.

120. Whitesell, J.D. et al. (2020) Regional, Layer, and Cell-Type-Specific Connectivity of the Mouse Default Mode Network. Neuron.

121. Saalmann, Y.B. et al. (2012) The pulvinar regulates information transmission between cortical areas based on attention demands. Science 337 (6095), 753-756.

122. Clemente-Perez, A. et al. (2017) Distinct thalamic reticular cell types differentially modulate normal and pathological cortical rhythms. Cell reports 19 (10), 2130-2142.

123. Steriade, M. and Deschenes, M. (1984) The thalamus as a neuronal oscillator. Brain Research Reviews 8 (1), 1-63.

124. Schmitt, L.I. et al. (2017) Thalamic amplification of cortical connectivity sustains attentional control. Nature 545 (7653), 219-223.

125. Bolkan, S.S. et al. (2017) Thalamic projections sustain prefrontal activity during working memory maintenance. Nature neuroscience 20 (7), 987.

126. Yantis, S. et al. (2002) Transient neural activity in human parietal cortex during spatial attention shifts. Nature neuroscience 5 (10), 995-1002.

127. Wimmer, R.D. et al. (2015) Thalamic control of sensory selection in divided attention. Nature 526 (7575), 705.

128. McAlonan, K. et al. (2006) Attentional modulation of thalamic reticular neurons. Journal of Neuroscience 26 (16), 4444-4450.

129. Qadir, H. et al. (2018) Structural Connectivity of the Anterior Cingulate Cortex, Claustrum, and the Anterior Insula of the Mouse. Front Neuroanat 12, 100.

130. Taylor, K.S. et al. (2009) Two systems of resting state connectivity between the insula and cingulate cortex. Human brain mapping 30 (9), 2731-2745.

131. Uddin, L.Q. (2015) Salience processing and insular cortical function and dysfunction. Nature reviews neuroscience 16 (1), 55-61.

132. Sridharan, D. et al. (2008) A critical role for the right fronto-insular cortex in switching between centralexecutive and default-mode networks. Proceedings of the National Academy of Sciences 105 (34), 1256912574.

133. Huang, Z. et al. (2021) Anterior insula regulates brain network transitions that gate conscious access. Cell Reports 35 (5), 109081.

134. Han, S.W. et al. (2019) Functional fractionation of the cingulo-opercular network: alerting insula and updating cingulate. Cerebral Cortex 29 (6), 2624-2638.

135. Lesh, T.A. et al. (2011) Cognitive control deficits in schizophrenia: mechanisms and meaning. Neuropsychopharmacology 36 (1), 316-338.

136. Wilcox, C.E. et al. (2014) Cognitive control in alcohol use disorder: deficits and clinical relevance. Reviews in the Neurosciences 25 (1), 1-24. 
137. Douglas, V.I. (1999) Cognitive control processes in attention deficit/hyperactivity disorder. In Handbook of disruptive behavior disorders, pp. 105-138, Springer.

138. Harvey, P.-O. et al. (2005) Cognitive control and brain resources in major depression: an fMRI study using the n-back task. Neuroimage 26 (3), 860-869.

139. Zeier, J.D. et al. (2012) Cognitive control deficits associated with antisocial personality disorder and psychopathy. Personality Disorders: Theory, Research, and Treatment 3 (3), 283.

140. Mathur, B.N. et al. (2009) Proteomic analysis illuminates a novel structural definition of the claustrum and insula. Cerebral Cortex 19 (10), 2372-2379.

141. Puelles, L. et al. (2016) Selective early expression of the orphan nuclear receptor Nr4a2 identifies the claustrum homolog in the avian mesopallium: impact on sauropsidian/mammalian pallium comparisons. Journal of Comparative Neurology 524 (3), 665-703.

142. Puelles, L. (2014) Development and evolution of the claustrum. In The Claustrum, pp. 119-176, Elsevier.

143. Bruguier, H. et al. (2020) In Search Of Common Developmental And Evolutionary Origin Of The Claustrum And Subplate. J Comp Neurol.

144. Sayalı, C. and Badre, D. (2019) Neural systems of cognitive demand avoidance. Neuropsychologia 123, 41-54.

145. Buzsáki, G. and Watson, B.O. (2012) Brain rhythms and neural syntax: implications for efficient coding of cognitive content and neuropsychiatric disease. Dialogues in clinical neuroscience 14 (4), 345.

146. Fries, P. (2005) A mechanism for cognitive dynamics: neuronal communication through neuronal coherence. Trends in cognitive sciences 9 (10), 474-480.

147. Salinas, E. and Sejnowski, T.J. (2000) Impact of correlated synaptic input on output firing rate and variability in simple neuronal models. Journal of neuroscience 20 (16), 6193-6209.

148. Sohal, V.S. et al. (2009) Parvalbumin neurons and gamma rhythms enhance cortical circuit performance. Nature 459 (7247), 698-702.

149. Morishima, Y. et al. (2009) Task-specific signal transmission from prefrontal cortex in visual selective attention. Nature neuroscience 12 (1), 85-91.

150. Herweg, N.A. et al. (2016) Theta-alpha oscillations bind the hippocampus, prefrontal cortex, and striatum during recollection: evidence from simultaneous EEG-fMRI. Journal of Neuroscience 36 (12), 3579-3587.

151. Mizuhara, H. et al. (2004) A long-range cortical network emerging with theta oscillation in a mental task. Neuroreport 15 (8), 1233-1238.

152. Mizuhara, H. et al. (2005) Long-range EEG phase synchronization during an arithmetic task indexes a coherent cortical network simultaneously measured by fMRI. Neuroimage 27 (3), 553-563.

153. Bächinger, M. et al. (2017) Concurrent tACS-fMRI reveals causal influence of power synchronized neural activity on resting state fMRI connectivity. Journal of Neuroscience 37 (18), 4766-4777.

154. Violante, I.R. et al. (2017) Externally induced frontoparietal synchronization modulates network dynamics and enhances working memory performance. Elife 6, e22001.

155. Cardin, J.A. et al. (2009) Driving fast-spiking cells induces gamma rhythm and controls sensory responses. Nature 459 (7247), 663-667. 


\section{Box 1. Testable Predictions of the NICC Model}

The NICC model yields specific structural and functional predictions to guide further investigation of the claustrum. A selection of these predictions is listed below. Some of these predictions are partially supported by available data, while others will serve as essential future tests of the model's veracity.

- Connectivity

- Individual claustrum neurons should preferentially project to different groups of cortical areas depending on the frontal inputs they receive. These targets should map onto areas identified as nodes of specific task-positive (e.g. FPN) and task-negative (e.g. DMN) cortical networks.

- Functional Circuits

○ Claustrum neuron activity should not encode sensory information but should encode aspects of task demand.

- Claustrum projection neurons should fire at the volitional initiation of internal reflection or another activity requiring DMN activation.

- Inputs capable of eliciting claustrum firing in physiological conditions should be from cortices associated with cognitive control (frontal regions).

- Cortical networks, cognition, and behavior

- Disruption of claustrum function at the onset of a task should impair the initiation, segregation and/or integrity of cortical networks.

- Degree of claustrum activation may scale with the integrity of the produced cortical network to meet task demands.[144]

- Behavior

- Selective suppression of different claustrum sub-circuits should elucidate the role of specific nodes within a network to behavior and network performance.

- Disruption of claustrum function should impair performance on tasks with high cognitive demand.

- Initiating cognitive control in humans should correlate with claustrum activation. 
o The claustrum should activate while switching between tasks that require different cortical network configurations and significant cognitive load demands.

\section{Box 2. From cortical synchrony to network emergence.}

Synchronous oscillations between different cortical areas are theorized to be vital for crossregional communication and necessary for cortical network performance.[145] This is because input from other cortical areas, synchronized with local oscillations, would be more likely to elicit action potentials than off-phase input,[146, 147] thereby decreasing cortical noise and amplifying signal.[148] As such, transmission of neural signals between non-synchronized regions is depressed, while transmission between synchronous regions is enhanced. This process allows for selective gating of information flow through the brain, as directly demonstrated by the ability, in humans, to direct top-down signals from prefrontal cortex to different posterior visual areas depending on the type of task being performed.[149]

Evidence indicates that increases in cortical synchrony are associated with increased network integrity. EEG signals of cortical oscillations correlate with functional connectivity and network strength across a variety of task-states.[150-152] Causally, synchronous transcranial alternating current (tACS) stimulation of left and right somatosensory cortex increases functional connectivity between those areas.[153] During a working memory task, power-synchronous tACS stimulation

of the middle frontal gyrus and inferior parietal lobule nodes (right FPN associated areas) enhances FPN activation in humans.[154] Induction of synchrony using tACS stimulation improves human performance on a working memory task,[154] while optogenetically driving fast-spiking interneurons in mouse barrel cortex induces theta synchrony and improves somatosensory discrimination.[155] These findings support the view that synchronized oscillatory activity supports cross-regional communication, cognitive network integrity, and performance on tasks that require multiple cognitive faculties. Thus, the ability to induce cortical synchrony for enhanced network integrity may support a cognitive control function. 


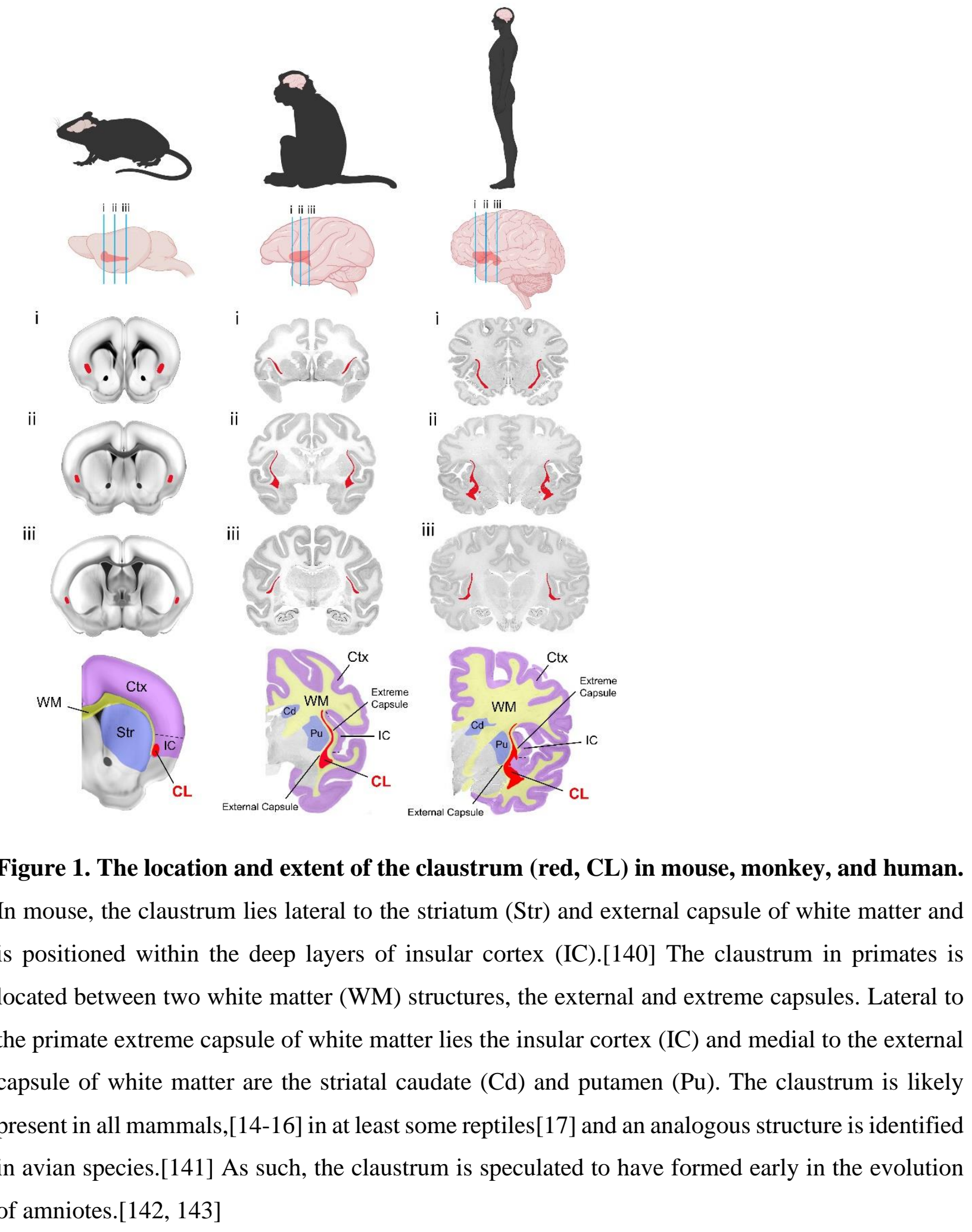




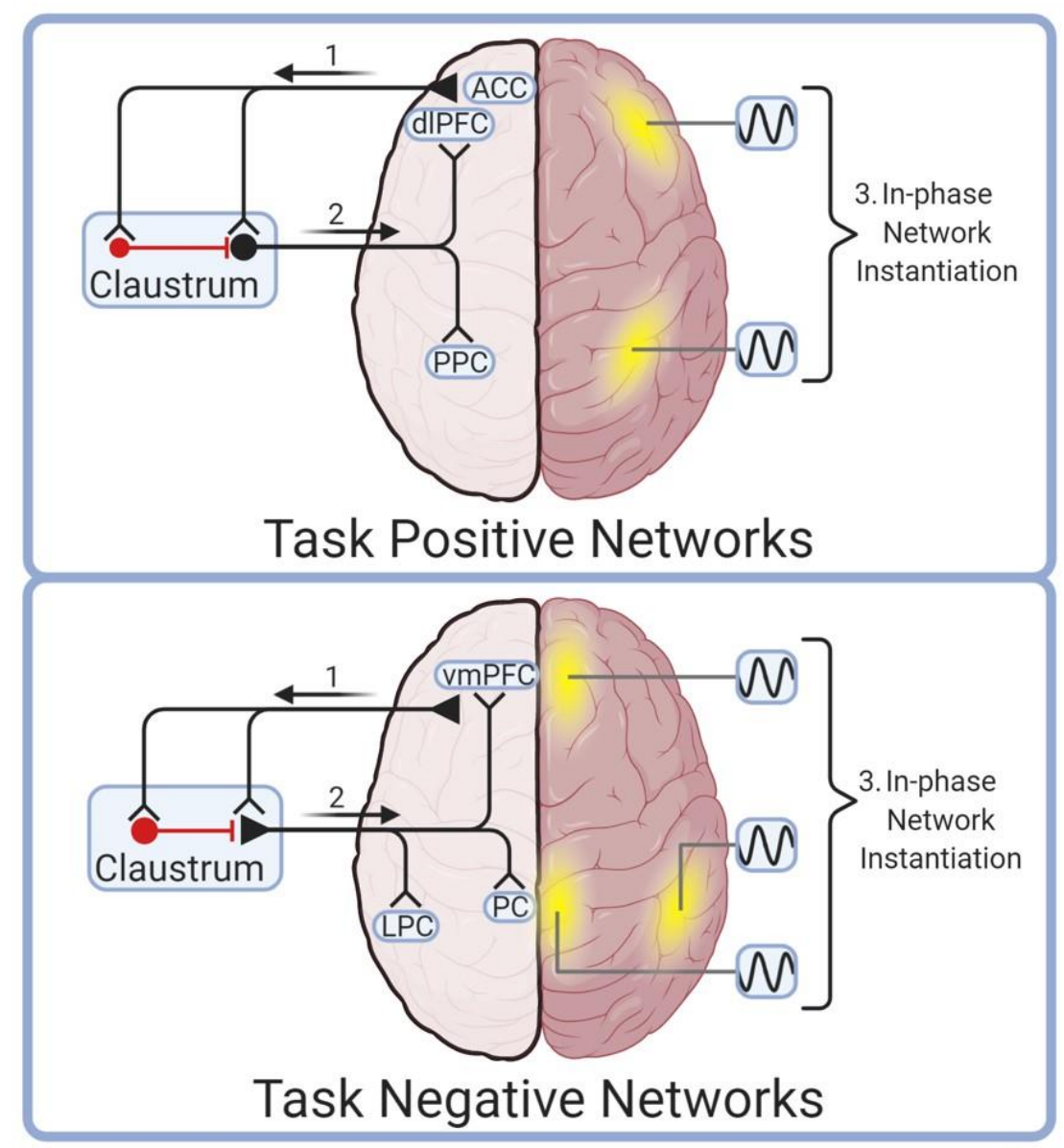

Figure 2. A summary of the NICC model. The NICC model proposes that frontal regions send cognitive control information to claustrum (1), which then transforms, amplifies, and broadcasts this information to cortical network nodes. (2) These cortical nodes are phase-locked by the claustro-cortical input to instantiate task-positive or task-negative cortical networks (3). 


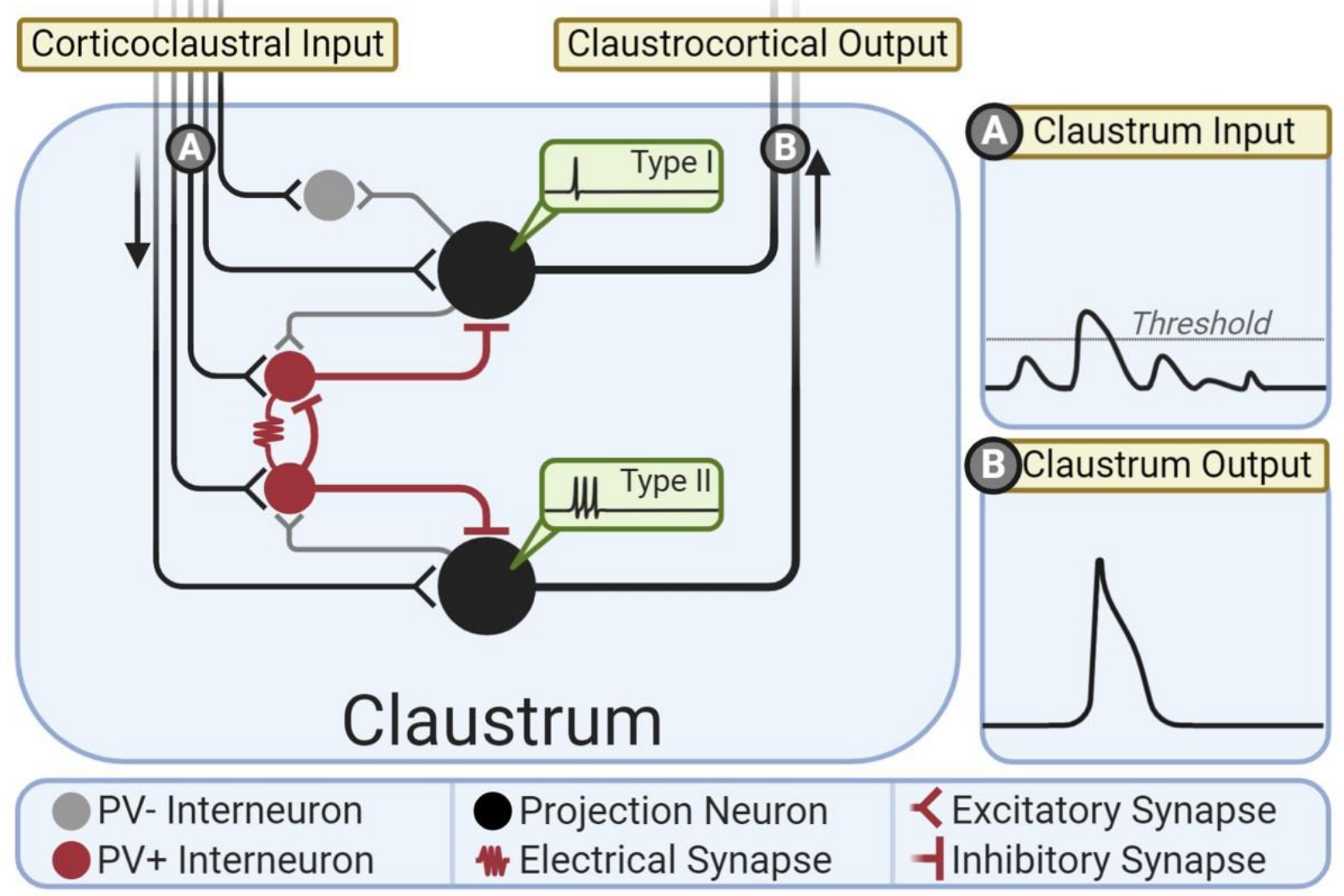

Figure 3. Cortico-claustro-cortical processing. Claustrum projection neurons are strongly inhibited by a tonically active and interconnected local parvalbumin-positive interneuron population (red). Claustrum projection neurons project predominantly to cortex with weaker innervation of neighboring claustrum interneurons; a subset of claustrum projection neurons burst fire in response to activation (Type II). The configuration of claustrum microcircuitry allows for sufficient cortical input (that which exceeds the "threshold" for claustrum inhibitory microcircuit control of claustrum projection neuron firing) to be transformed and amplified for claustro-cortical broadcast $(\mathbf{B})$. 


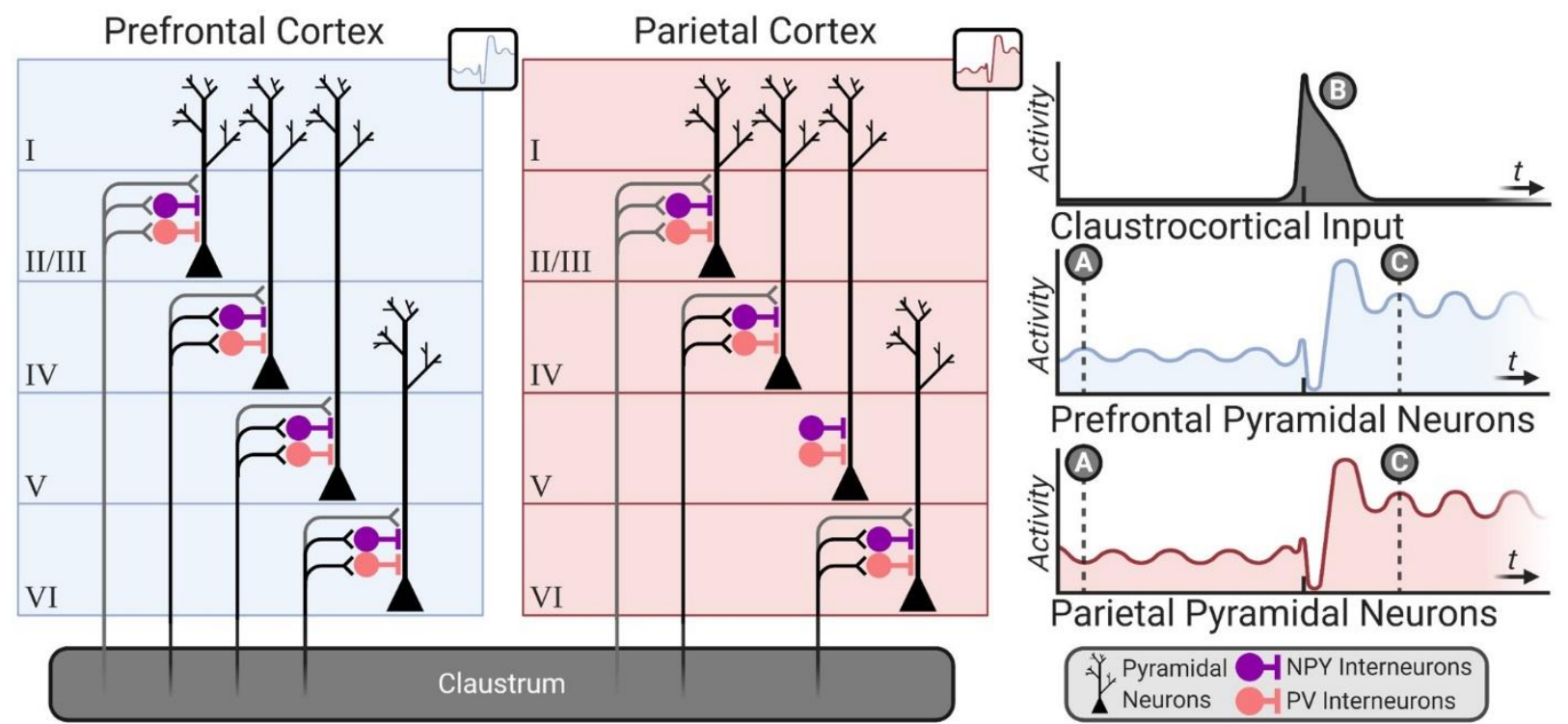

Figure 4. Claustrum innervation of cortex and the effects of claustro-cortical input on cortical activity. Claustrum afferents are biased toward deeper layers of cortex and to neuropeptide Y-expressing interneurons. The exception to this is a lack of claustro-cortical input to layer $\mathrm{V}$ parietal cortex in mouse, ${ }^{[88]}$ which likely reflects a lack of claustrum control of layer $\mathrm{V}$ neurons projecting to dorsolateral striatum/putamen. Task-positive cortical network components (eg., prefrontal cortex and parietal cortex) at cognitive rest display relatively desynchronized oscillations (A). Claustrum firing (B) initially elicits strong inhibition of pyramidal neurons via activation of parvalbumin-expressing and neuropeptide $\mathrm{Y}$-expressing interneurons, followed by resumption of pyramidal neuron excitation mediated, in part, by direct claustro-cortical input. This induces a phase-locking of activity across cortical regions, from which subsequent oscillatory frequencies may be governed by cortico-cortical and thalamocortical circuits. With disparate cortical regions now participating in phase-locked activity, a synchronized network is instantiated. (C). 